\title{
Shape-sensing robotic-assisted bronchoscopy for pulmonary nodules: initial multicenter experience using the lon Endoluminal System
}

Michael J. Simoff ${ }^{* *}$, Michael A. Pritchett ${ }^{2,3}$, Janani S. Reisenauer ${ }^{4}$, David E. Ost ${ }^{5}$, Adnan Majid ${ }^{6}$, Colleen Keyes ${ }^{7}$, Roberto F. Casal ${ }^{5}$, Mihir S. Parikh' ${ }^{6}$, Javier Diaz-Mendoza ${ }^{1}$, Sebastian Fernandez-Bussy ${ }^{8}$ and Erik E. Folch ${ }^{7}$

\begin{abstract}
Background: Traditional bronchoscopy provides limited approach to peripheral nodules. Shape-sensing roboticassisted bronchoscopy (SSRAB, Ion ${ }^{\text {TM }}$ Endoluminal System) is a new tool for minimally invasive peripheral nodule biopsy. We sought to answer the research question: Does SSRAB facilitate sampling of pulmonary nodules during bronchoscopists' initial experience?
\end{abstract}

Methods: The lead-in stage of a multicenter, single-arm, prospective evaluation of the lon Endoluminal System (PRE$\mathrm{ClsE}$ ) is described. Enrolled subjects $\geq 18$ years old had recent computed tomography evidence of one or more solid or semi-solid pulmonary nodules $\geq 1.0$ to $\leq 3.5 \mathrm{~cm}$ in greatest dimension and in any part of the lung. Subjects were followed at 10- and 30-days post-procedure. This stage provided investigators and staff their first human experience with the SSRAB system; safety and procedure outcomes were analyzed descriptively. Neither diagnostic yield nor sensitivity for malignancy were assessed in this stage. Categorical variables are summarized by percentage; continuous variables are summarized by median/interquartile range (IQR).

Results: Sixty subjects were enrolled across 6 hospitals; 67 nodules were targeted for biopsy. Median axial, coronal and sagittal diameters were $<18 \mathrm{~mm}$ with a largest cardinal diameter of $20.0 \mathrm{~mm}$. Most nodules were extraluminal and distance from the outer edge of the nodule to the pleura or nearest fissure was $4.0 \mathrm{~mm}$ (IQR: 0.0, 15.0). Median bronchial generation count to the target location was 7.0 (IQR: 6.0, 8.0). Procedure duration (catheter-in to catheterout) was $66.5 \mathrm{~min}$ (IQR: 50.0, 85.5). Distance from the catheter tip to the closest edge of the virtual nodule was $7.0 \mathrm{~mm}$ (IQR: 2.0, 12.0). Biopsy completion was $97.0 \%$. No pneumothorax or airway bleeding of any grade was reported.

Conclusions: Bronchoscopists leveraged the Ion SSRAB's functionality to drive the catheter safely in close proximity of the virtual target and to obtain biopsies. This initial, multicenter experience is encouraging, suggesting that SSRAB may play a role in the management of pulmonary nodules.

Clinical Trial Registration identifier and date NCT03893539; 28/03/2019.

\footnotetext{
*Correspondence: MSimoff1@hfhs.org

${ }^{1}$ Bronchoscopy and Interventional Pulmonology, Lung Cancer Screening

Program, Department of Pulmonary and Critical Care Medicine, Henry

Ford Hospital, Wayne State University School of Medicine, 2799 West

Grand Blvd, Detroit, MI 48202, USA

Full list of author information is available at the end of the article
}

(c) The Author(s) 2021. Open Access This article is licensed under a Creative Commons Attribution 4.0 International License, which permits use, sharing, adaptation, distribution and reproduction in any medium or format, as long as you give appropriate credit to the original author(s) and the source, provide a link to the Creative Commons licence, and indicate if changes were made. The images or other third party material in this article are included in the article's Creative Commons licence, unless indicated otherwise in a credit line to the material. If material is not included in the article's Creative Commons licence and your intended use is not permitted by statutory regulation or exceeds the permitted use, you will need to obtain permission directly from the copyright holder. To view a copy of this licence, visit http://creativecommons.org/licenses/by/4.0/. The Creative Commons Public Domain Dedication waiver (http://creativecommons.org/publicdomain/zero/1.0/) applies to the data made available in this article, unless otherwise stated in a credit line to the data. 
Keywords: Pulmonary nodules, Biopsy, Shape sensing, Bronchoscopy, Robotic assistance, lon

\section{Background}

Chest imaging has seen significant growth in the United States and worldwide due to its non-invasive ability to detect pulmonary conditions. In the United States more than an estimated 1.6 million nodules are detected each year as incidental findings on chest radiographs and computed tomography (CT) scans [1]. Also seen is increased identification of nodules due to the growth in lung cancer screening programs based on low-dose CT scans to assess patients who are high risk for lung cancer. Such screening has resulted in an $8-51 \%$ incidence of solitary pulmonary nodules within selected populations [2]. While most nodules may require surveillance, a significant number require tissue biopsy.

Because of their larger diameters, standard bronchoscopes cannot progress beyond the subsegmental bronchi and consequently provide a limited approach to peripheral nodules [3]. The overall sensitivity for malignancy of flexible bronchoscopy is $34 \%$ for lesions $<2 \mathrm{~cm}$ and $63 \%$ for $>2 \mathrm{~cm}$ [4]. The development of virtual bronchoscopy, fluoroscopic guidance, radial endobronchial ultrasound (rEBUS), ultrathin bronchoscopes, and electromagnetic navigation bronchoscopy (ENB) for the diagnosis of peripheral nodules has improved our ability to sample smaller and more peripheral lesions. Yet, a meta-analysis resulted in a pooled diagnostic yield (based on these techniques prior to 2010) evidenced $60.9 \%$ for lesions $\leq 2 \mathrm{~cm}$-demonstrating the continued challenge to approaching small peripheral nodules $[5,6]$. Two recent studies-a real-world single-arm cohort and a meta-analysis, respectivelydescribed a 73\% yield from ENB for nodules with a median size of $2 \mathrm{~cm}$ and a pooled sensitivity for malignancy of $77 \%$ with a good safety profile with an average lesion size of $23.2 \mathrm{~mm}$ [7, 8]. Such reports highlight the need for improved procedural outcomes for small peripheral nodules by developing new bronchoscopy tools and approaches, while maintaining a low complication rate.

A shape-sensing robotic-assisted bronchoscope (SSRAB) is a new tool for minimally invasive peripheral nodule biopsy. The Ion ${ }^{\mathrm{TM}}$ Endoluminal System (Intuitive Surgical, Inc., Sunnyvale, CA) received its $510 \mathrm{~K}$ from the FDA in 2019. In the first human use study of a precommercial iteration of the Ion Endoluminal System, targets with a mean size of approximately $14 \mathrm{~mm}$ were reached in $96.6 \%$ of cases; the overall diagnostic yield was $79.3 \%$ with no reported incidence of pneumothorax or bleeding [9].
Does SSRAB facilitate sampling of pulmonary nodules in human subjects during bronchoscopists' early experience? The authors hypothesize that SSRAB does facilitate biopsy of pulmonary nodules, including small and peripherally based nodules, in these subjects. This manuscript is a report of the initial multicenter experience in human subjects, including procedural characteristics and descriptive outcomes. [ClinicalTrials.gov identifier and date: NCT03893539; 28/03/2019].

\section{Methods}

The current study describes the initial or lead-in stage (Stage 0) of the larger PRECIsE study-a multistage, single-arm, prospective evaluation of the SSRAB System to bronchoscopically approach and facilitate sampling of pulmonary nodules. The purpose of this lead-in stage was to provide participating investigators and support staff their first human experience with the SSRAB system and its associated workflow. The study period (date of first enrollment to last follow up) was from $29 \mathrm{March}$, 2019 to 10 January, 2020. For the lead-in cases, subjects were followed to and not beyond 30 days post procedure. The focus was collection of early complications and safety data. The performance metrics of yield and sensitivity will be addressed and analyzed in subsequent publications.

Pre-specified enrollment in this initial stage was limited to 10 subjects per each of the six participating centers, with at least 5 subjects per bronchoscopist. Enrolled subjects were $\geq 18$ years old, were suitable candidates for elective bronchoscopy, had recent evidence on CT of one or more solid or semi-solid pulmonary nodules $\geq 1.0$ to $\leq 3.5 \mathrm{~cm}$ in greatest dimension and in any part of the lung. Subjects considered for enrollment in this study had a moderate-to-high risk of malignancy; high-risk subjects were enrolled if they wanted diagnostic confirmation prior to treatment. Subjects with a suspicion of metastatic disease were also considered; all considered subjects included those for whom investigators would consider further interventions if indicated to confirm diagnosis. Each center obtained institutional review board (IRB) approval (study sites, IRB committee names, and approval numbers follow. Mayo Clinic: Mayo Clinic IRB 18-011348; First Health Moore Regional Hospital: Western IRB 20183121; Massachusetts General Hospital: Dana Farber Cancer Institute IRB 19-209; Beth Israel Deaconess Medical Center: Dana Farber Cancer Institute IRB 19-209; Henry Ford Hospital: Henry Ford Health System IRB 12822; University of Texas MD 
Anderson Cancer Center: MD Anderson Cancer Center IRB IRB00006023). Enrolled study participants provided written informed consent, and study subject confidentiality was maintained according to the Health Insurance Portability and Accountability Act requirements.

Briefly described, the SSRAB system is comprised of a robotic system cart, controller and fully articulating catheter instrument with embedded shape-sensing capabilities (Fig. 1). The robotic system cart facilitates the movement of the catheter instrument via the instrument arm by translating input from the standalone controller and leverages a pull-wire system to drive the catheter into the airways under direct visualization provided by a vision probe. The cart houses two system monitors that provide visual information including: virtual and live airway views, the airway tree with catheter position overlaid, target information, and third-party video sources such as radial endobronchial ultrasound (rEBUS)and fluoroscopy (Figs. 2 and 3). The fully articulating catheter is $3.5 \mathrm{~mm}$ in outer diameter with a 2-mm working channel and a steerable distal tip, which can be articulated up to 180 degrees in any direction (Fig. 4). A thin flexible fiber, which provides the basis for the shape-sensing technology, is embedded along the catheter's entire length

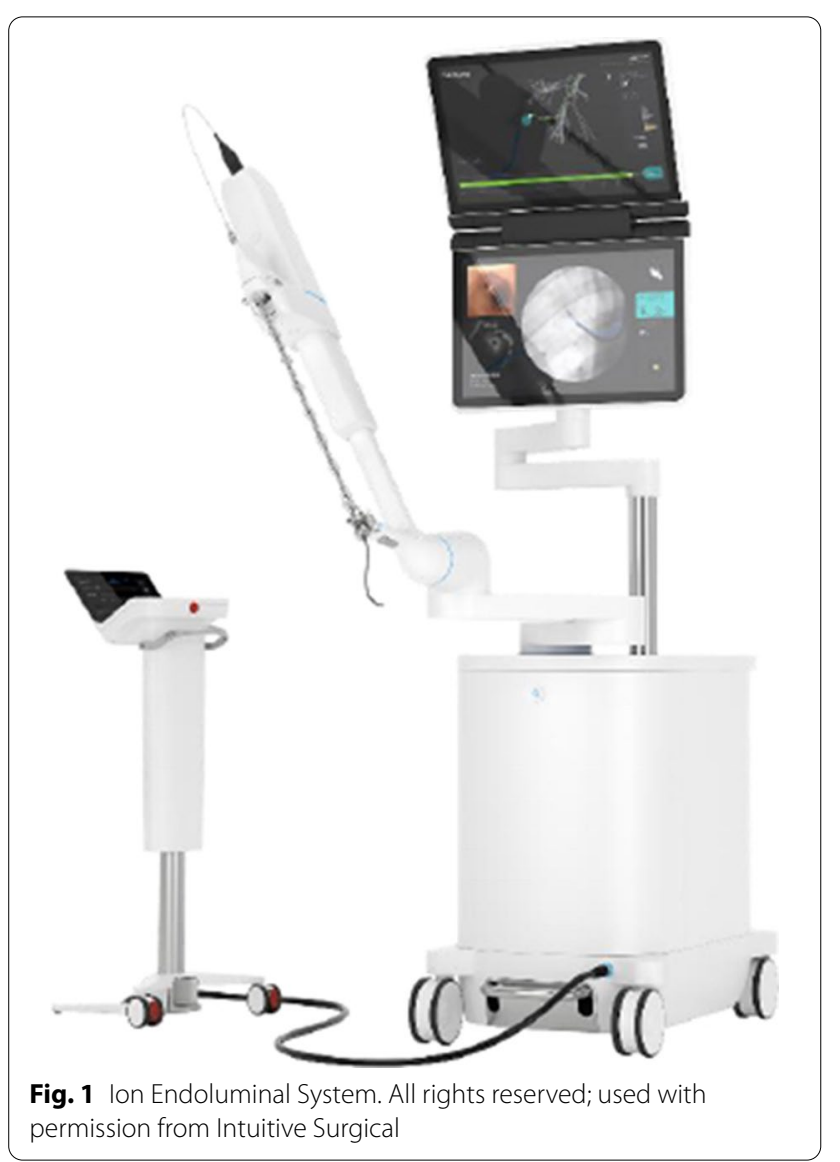

and measures its own shape hundreds of times per second, representing and displaying throughout the procedure the shape and position of the catheter relative to the anatomy (Fig. 5). The catheter also provides feedback to the robotic control algorithm that maintains the intended position for the catheter instrument, enabling a fixed and stable position and correcting for any deflections from the intended position by providing extra force through the appropriate pull-wire system. The technology and instruments are immune to electromagnetic interference and are unaffected by metallic objects or electromagnetic fields.

Each investigator completed standardized training, which allowed bronchoscopists to gain experience and basic familiarity with the system through in-depth dry lab in-service training, a porcine lab, and a cadaveric lab. In-service training was completed prior to any clinical cases primarily for support staff and included assistance with room setup and process workflow.

Pre-procedure CT scans $(0.75-1.25 \mathrm{~mm}$ slice thickness) were segmented using the system-specific PlanPoint $^{\mathrm{TM}}$ planning software with pathway planning completed and reviewed through a virtual simulation (Fig. 6a and Fig. 6b). With the patient under general anesthesia, standard airway examinations were initially performed using a flexible bronchoscope and included clearing of secretions and review of the normal anatomy to be navigated. The SSRAB system was then docked to the endotracheal tube via a magnetic adapter. After registration, navigation of the catheter to the target nodule was completed under direct visualization and in accordance with the virtual plan created using the pre-procedure CT. When the target was reached, the vision probe was removed and a combination of rEBUS and fluoroscopy were used to assess real-time information regarding the nodule and to refine the biopsy location. After appropriate adjustments were made to the catheter position, sampling commenced using the system-specific flexible needle (Flexision ${ }^{\mathrm{TM}}, 19 \mathrm{G}, 21 \mathrm{G}$, or 23G) using the cloud biopsy technique. Cloud biopsy, which describes the systematic and consistent placement and/or redirection of biopsy tools in specific sampling areas, was enabled by the fine manipulation of the scope tip in any plane to optimize the angle towards the target for further biopsies. The cloud biopsy facilitates the collection of samples from four different quadrants of a target nodule. The bronchoscopist was able to perform this technique due to the ability to make micro adjustments of the catheter tip to optimize the biopsy zone. Forceps, cytology brush, and/or bronchioalveolar lavage were used at the bronchoscopist's discretion. Rapid on-site cytology evaluation (ROSE) was available at 5 of 6 participating centers and was used according to institution practice and was not 


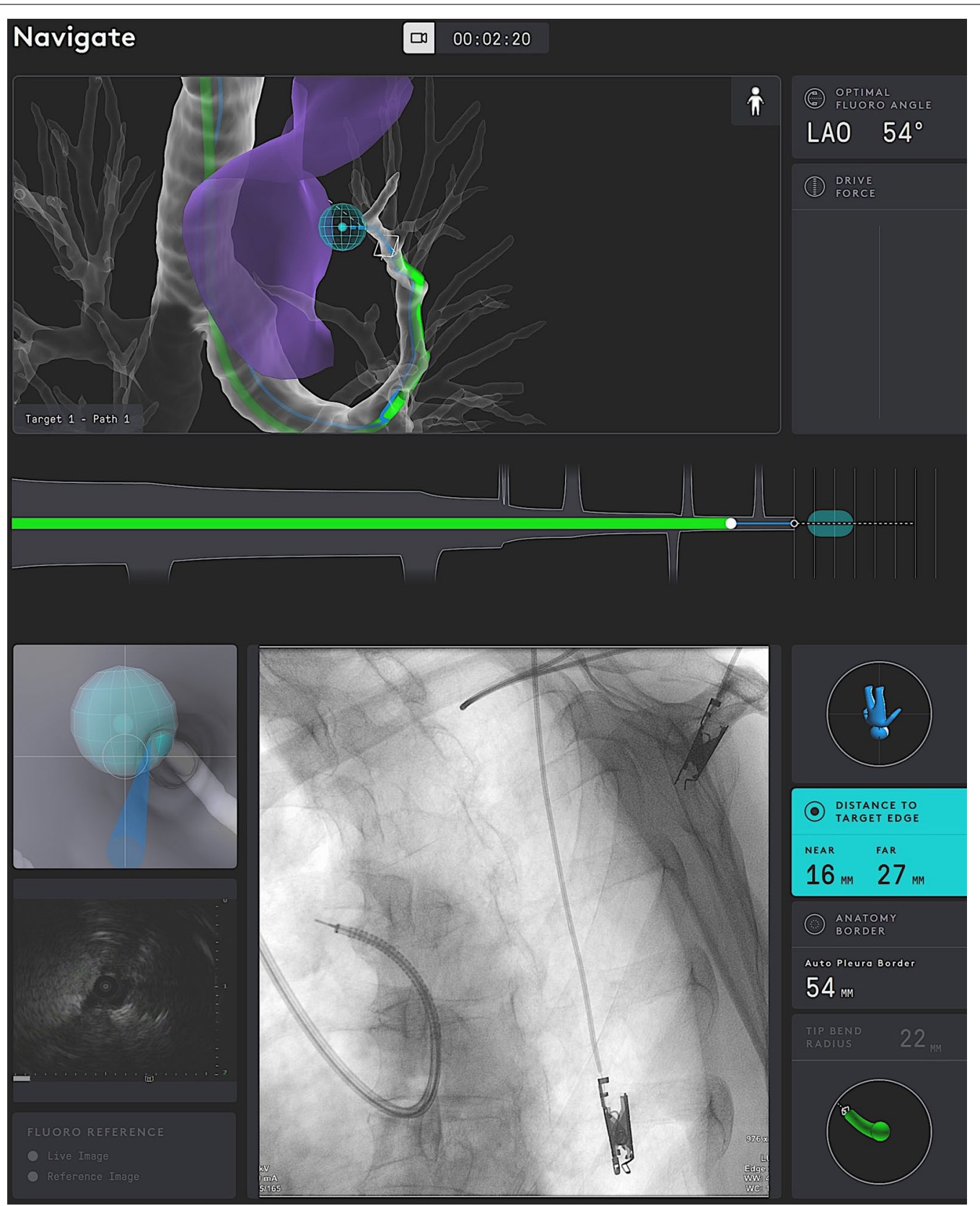

Fig. 2 Procedural screenshot from one case. Top monitor screens: virtual airway view showing catheter within airway tree; blue ball represents target; green subway view at bottom shows the progress of the catheter to the target; drive force displayed on the right. Bottom monitor screens: (upper left) virtual target with target view; (bottom left) integrated EBUS view; (center) fluoroscopy view of catheter with tool extension; (right side) informational screen displaying distance to virtual target, anatomy borders, orientation guide, and catheter tip bend radius

standardized. Following the procedure, a fluoroscopic check for pneumothorax was performed; in the same anesthesia event, EBUS-TBNA staging (where indicated) was performed. A chest $\mathrm{x}$-ray was taken at least one-hour post-procedure to assess for delayed pneumothorax.

Both the nodule size (assessed in axial, coronal and sagittal planes) as well as the distance of the closest edge of the target nodule to the closest pleura or fissure were both based on pre-procedure CT scans. Bronchial generation count was assessed with the trachea representing generation 0 and each subsequent carina or bifurcation/ trifurcation counting as an additional generation based on the pre-procedure $\mathrm{CT}$ and typically using the segmented model. Each bronchoscopist assessed the nodule 


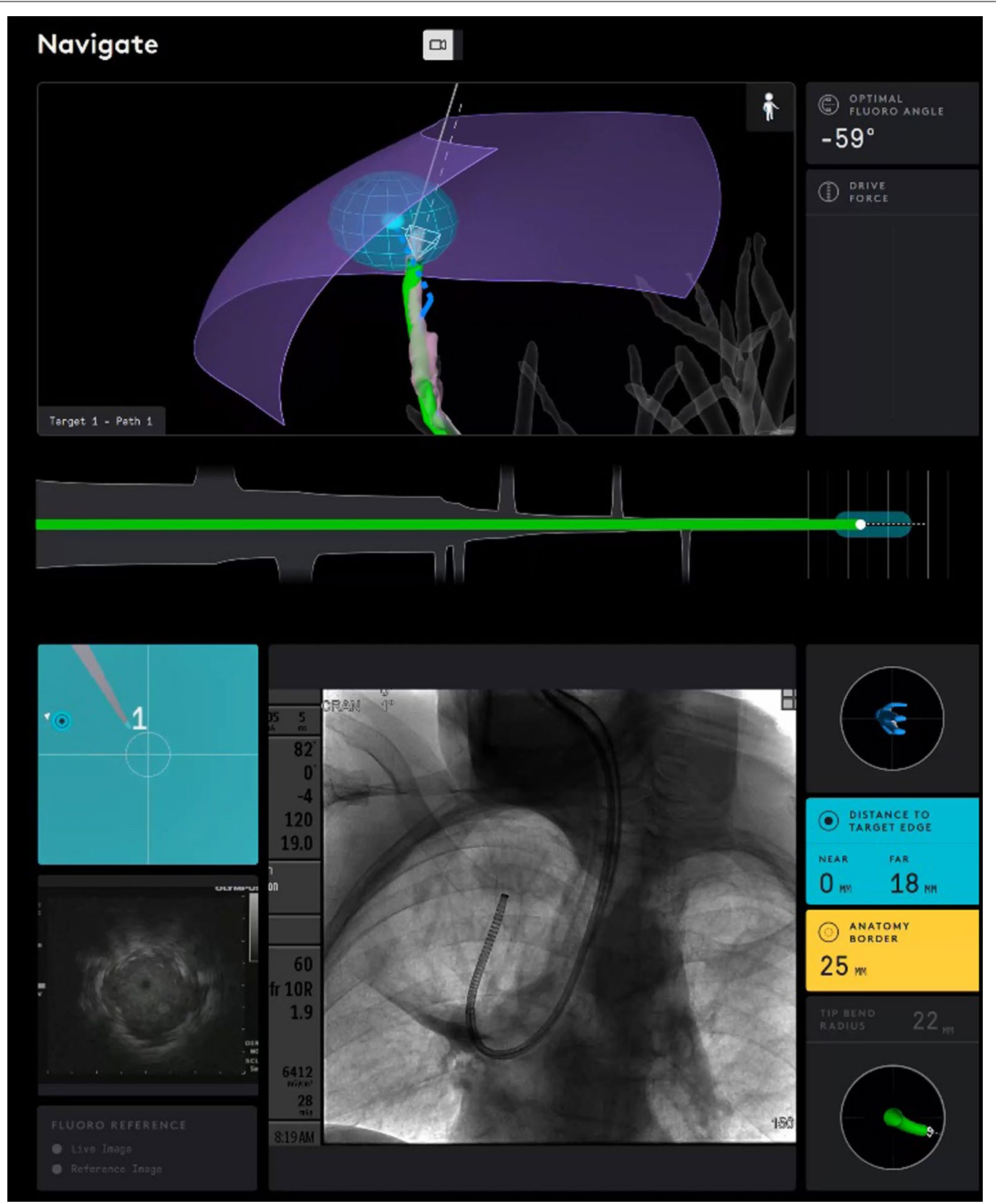

Fig. 3 Another screenshot from a case showing (top) the catheter reaching the virtual target and (bottom) fluoroscopic view of catheter bend to the apical lesion

characteristics including presence of bronchus sign on pre-procedure CT and rEBUS visualization during the procedure according to individual practice. Registration time was the total time necessary to complete registration prior to the start of navigation; biopsy time (using SSRAB for the peripheral nodule) was the time from first biopsy tool insertion to last peripheral biopsy tool withdrawn. Procedure time specifically for the SSRAB system was measured from time of catheter insertion to withdrawal; durations of airway survey prior to the use of Ion nor the duration of further diagnostic procedures, including EBUS-TBNA, were not included in these times. Use of a PlanPoint-generated path reflected whether the bronchoscopist followed a system-generated pathway to the virtual target nodule. Catheter positions represented the unique parked locations of the catheter tip in relation to the nodule. Tool passes reflected the total number of biopsy tools passed through the catheter, with each tool 


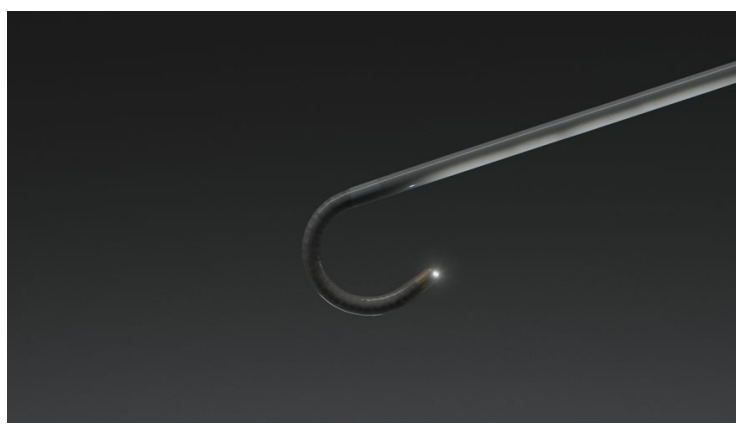

Fig. 4 Catheter instrument (3.5 mm outer diameter) with vision probe in articulated position. All rights reserved; used with permission from Intuitive Surgical

counted separately and no reliance on sample acquisition. Distance between the catheter tip and the closest edge of the virtual target when the catheter was parked prior to biopsy was displayed on the system cart monitor. Adverse events related to the bronchoscopy procedure were collected, including any type of pneumothorax and bleeding that did not stop spontaneously or that required intervention. Subjects were followed at 10 days and 30 days post-procedure.

The purpose of this stage of the study was to provide participating investigators and support staff their first human experience with the SSRAB system; safety and procedure outcomes were analyzed. Diagnostic yield or sensitivity for malignancy were not assessed in this stage. Data in the current analysis will not be used in subsequent multicenter outcomes analyses. Subject and procedure outcomes for this lead-in stage are presented descriptively; categorical variables are summarized by percentage; continuous variables are summarized by median/IQR. The statistical software package $\mathrm{SAS}^{\circledR}$ version 9.4 (SAS Institute, Cary, NC, USA) was used.

\section{Results}

Sixty subjects were enrolled across the six participating institutions, and a total of 67 nodules were targeted for biopsy. The study protocol recommended the timing between the pre-procedure CT scan and the procedure day not to exceed 21 days. On average, the CTs were taken approximately 5 days prior to the procedures and approximately $30 \%$ of the enrolled subjects had their CT scans the day of their procedures. Demographics are described in Table 1 . The median pretest probability of malignancy using the Mayo/Swensen model was $46.3 \%$ (IQR: $23.8,77.3)$ [9]; the majority $(76.7 \%)$ of subjects were classified as American Society of Anesthesiologists (ASA) III.

Nodule characteristics are provided in Table 2. Median axial, coronal, and sagittal dimensions were each 17.5, 16.0 , and $16.2 \mathrm{~mm}$, respectively, and the median largest cardinal diameter was $20.0 \mathrm{~mm}$. Approximately half $(52.3 \%)$ of nodules were located in the upper lobes and $82.1 \%$ of nodules were solid. Nodules were predominately extraluminal as assessed visually and the median distance from the outer edge of the nodule to the nearest pleura or fissure was $4.0 \mathrm{~mm}$ (IQR: 0.0, 15.0), with approximately $31 \%$ in contact with the pleura or fissure as measured on the pre-procedure CT scan. A bronchus sign was present for $32.7 \%$ of nodules. Median bronchial generation count was 7.0 (IQR: 6.0, 8.0).

Procedural characteristics, including the median duration of each stage of the procedure, are provided (Table 3 ). Navigation planning pre-procedure was $10.0 \mathrm{~min}$ (IQR: $5.0,15.0)$, and the median duration of the procedures from catheter in-to catheter out was $66.5 \mathrm{~min}$ (IQR: 50.0, 85.5) including sampling of multiple nodules within the same subject ( 7 cases, each with 2 nodules biopsied) as well time associated with ROSE results and/or obtaining multiple samples. ROSE was completed in $78.3 \%$ of cases.

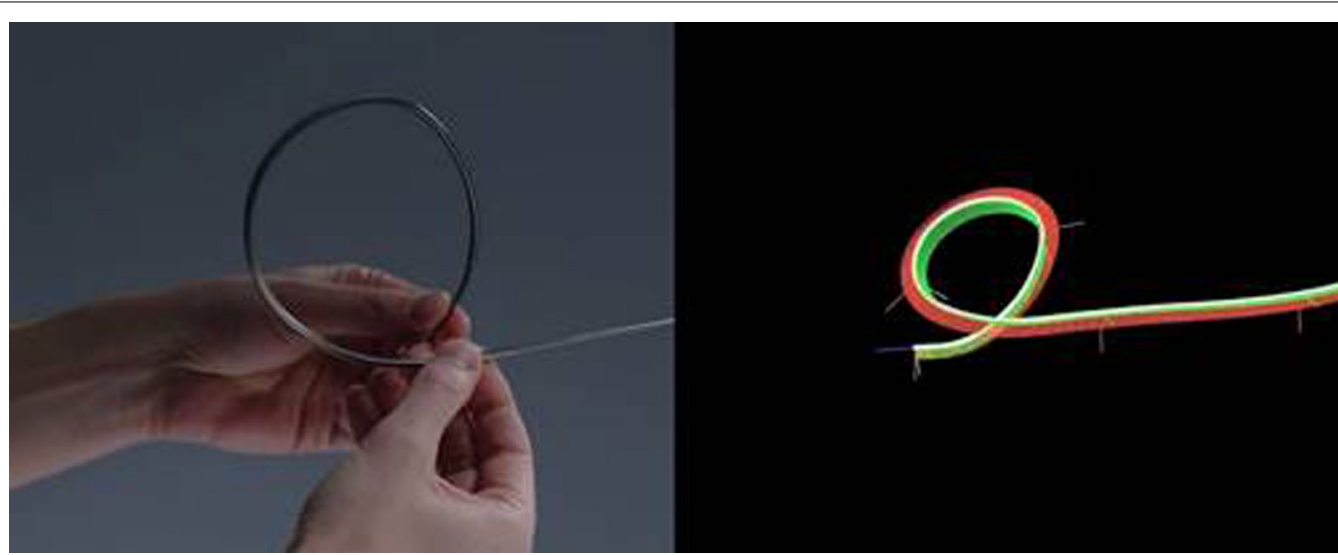

Fig. 5 Image illustrating shape-sensing technology along catheter's entire length. All rights reserved; used with permission from Intuitive Surgical 


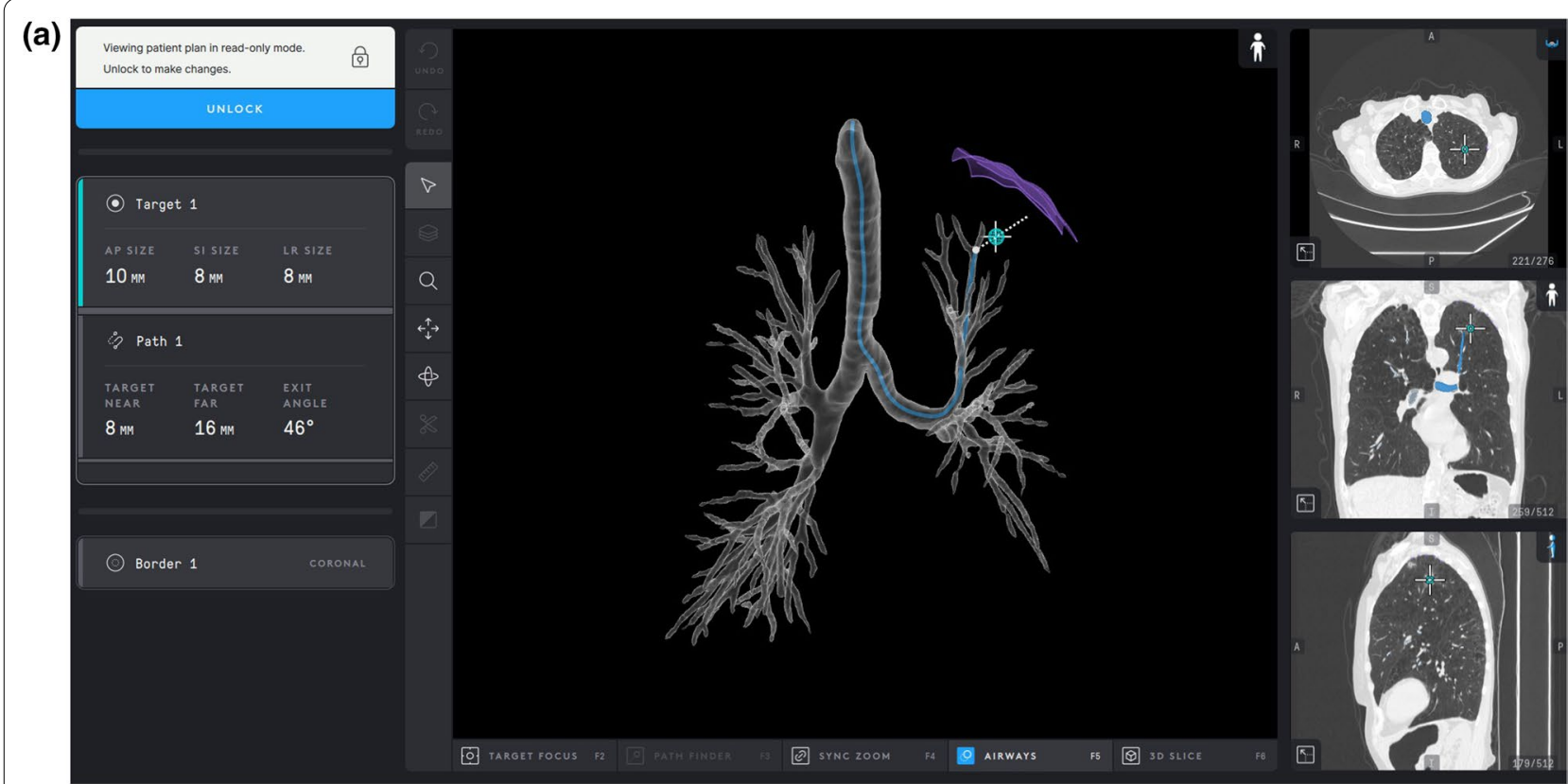

(b)

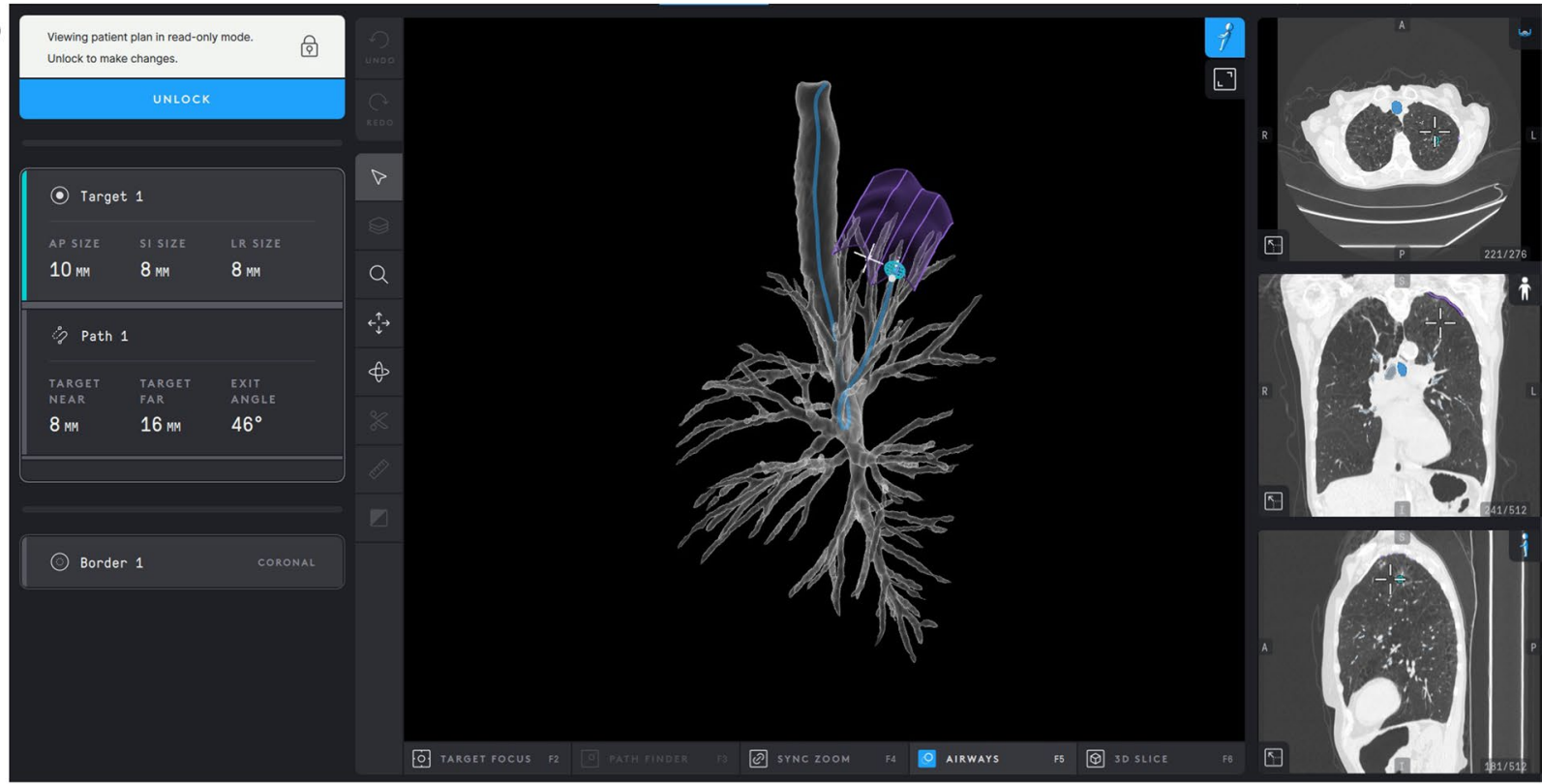

Fig. 6 a, b Segmented CT scans of the same subject from PlanPoint planning software from two orientations, both of which show the target nodule, identified pathway, and anatomy border

Once registration was completed, navigation to the first nodule took $5.0 \mathrm{~min}$ (IQR: 3.0, 10.0). In nearly all (93.1\%) of the cases, the PlanPoint path was used to reach the target nodule. During the biopsy sequence using a needle first, forceps, and/or cytology brush, there was an average of four catheter tip positions. There was a median of 13 biopsy tool passes per procedure, and rEBUS was used for 66 out of 67 nodules. rEBUS was not attempted in two cases due to technical due to technical or clinical reasons at the discretion of the proceduralist. The remaining five nodules were in the lower lobe, and three did not have CT bronchus sign. The median time to achieve first rEBUS visualization was 8 min from navigation start. The use of rEBUS achieved visualization of $89.4 \%$ of nodules where rEBUS was attempted; of nodules visualized with rEBUS, an initial concentric view was obtained in $39 \%$ 
Table 1 Baseline characteristics of subjects ( $n=60$ subjects)

\begin{tabular}{ll}
\hline Variable & Outcome \\
\hline Age, median, y (IQR) & $70.7(63.1,76.6)$ \\
BMI, median kg/m² (IQR) & $25.0(22.9,31.6)$ \\
Gender, n (\%) & \\
Female & $35(58.3)$ \\
Male & $25(41.7)$ \\
Pretest probability of malignancy, median \% (IQR) ${ }^{\text {a }}$ & $46.3(23.8,77.3)$ \\
ASA class, n (\%) & \\
॥ & $14(23.3)$ \\
III & $46(76.7)$ \\
\hline
\end{tabular}

$I Q R$ interquartile range, $B M I$ body mass index, $A S A$ American Society of Anesthesiologists

a Swensen's Formula: low risk < 5\%; indeterminate risk 5-65\%; high risk >65\%; from reference [9]

Table 2 Nodule characteristics ( $n=67$ nodules)

\begin{tabular}{|c|c|}
\hline Variable & Outcome \\
\hline \multicolumn{2}{|l|}{ Size, median mm (IQR) } \\
\hline Axial & $17.5(12.0,24.0)$ \\
\hline Coronal & $16.0(11.8,21.0)$ \\
\hline Sagittal & $16.2(12.0,22.0)$ \\
\hline Largest cardinal diameter & $20.0(14.0,27.0)$ \\
\hline \multicolumn{2}{|l|}{ Lobe location, n (\%) } \\
\hline LLL & $8(11.9)$ \\
\hline LUL & $18(26.9)$ \\
\hline RLL & $21(31.3)$ \\
\hline RML & $3(4.5)$ \\
\hline RUL & $17(25.4)$ \\
\hline Distance from pleura or fissure, median mm (IQR) & $4.0(0.0,15.0)$ \\
\hline Bronchial generation count, median n (IQR) & $7.0(6.0,8.0)$ \\
\hline \multicolumn{2}{|l|}{ Location, n (\%) } \\
\hline Endoluminal & $10(15.4)$ \\
\hline Extraluminal & $55(84.6)$ \\
\hline \multicolumn{2}{|l|}{ Nodule type, n (\%) } \\
\hline Solid & $55(82.1)$ \\
\hline Cavitary & 4 \\
\hline Semi-solid & $12(17.9)$ \\
\hline CT Bronchus sign present, n (\%) & $25(37.3)$ \\
\hline rEBUS attempted, n (\%) & $66(98.5)$ \\
\hline rEBUS visualization, n (\%) & $59(89.4)$ \\
\hline Concentric, initially & $23(40)$ \\
\hline Eccentric converted to concentric & $8(13.6)$ \\
\hline Eccentric & $28(47.5)$ \\
\hline
\end{tabular}

$I Q R$ interquartile range, $L L L$ left lower lobe, $L U L$ left upper lobe, $R L L$ right lower lobe, $R M L$ right middle lobe, $R U L$ right upper lobe, $r E B U S$ radial endobronchial ultrasound

a 2 subjects had early termination

${ }^{\mathrm{b}} \mathrm{rEBUS}$ visualization is based on nodules attempted
Table 3 Procedural characteristics ( $n=60$ subjects)

\begin{tabular}{ll}
\hline Variable & Result \\
\hline Duration, median min (IQR) & \\
Navigation planning & $10.0(5.0,15.0)$ \\
Procedure (scope in to scope out) & $66.5(50.0,85.5)$ \\
Registration & $8.5(5.0,14.0)$ \\
Navigation to 1st nodule & $5.0(3.0,10.0)$ \\
Biopsy completion & $31.5(25,46.5)$ \\
Lymph node staging performed, n (\%) & $47(78.3)$ \\
PlanPoint path used, n (\%) & $54(93.1)$ \\
Time to 1st rEBUS visualization, min (IQR) & $8.00(4.0,13.5)$ \\
Catheter positions, n (IQR) & $4.0(2.0,7.0)$ \\
Tool passes, median n (IQR) & $13.0(8.0,15.0)$ \\
Tools used, n (\%), & \\
Needlec & $58(100)$ \\
Forceps & $40(69)$ \\
Brush & $28(48.3)$ \\
\hline
\end{tabular}

IQR interquartile range

${ }^{a}$ Tools used out of 58 completed procedures; 2 subjects with early termination not included

${ }^{b}$ More than one tool may have been used per case

' System-specific Flexision needle

of visualized nodules, while an additional $13.6 \%$ of nodules that were initially found to have an eccentric view became concentric after further adjustment and creation of a path to the nodule.

Biopsy completion, whereby a tool was passed through the catheter and a tissue sample obtained, was $96.7 \%$ for all subjects and $97.0 \%$ for all nodules (Table 4). Among completed biopsies, the median distance from the catheter tip to the edge of the virtual target was $7.0 \mathrm{~mm}$ (IQR: $2.0,12.0)$. There were two subject cases where biopsies were not attempted and an alternative method was used. In one case, the CT slice thickness was incompatible for registration; the case was completed using bronchoscopy with rEBUS. In the other case, the physician was unable to reach the nodule due to the lack of a patent airway and the procedure was completed using an esophageal approach using a cEBUS scope (EUS-B).

No serious adverse events were reported, including no pneumothorax or airway bleeding of any grade $[10,11]$. Two complications were reported, both of which were classified as Common Terminology Criteria for Adverse Event (CTCAE) grade 2 [12]. One subject experienced cardiac arrhythmia during the procedure, which was treated with medication with anesthesia and resolved immediately. The subject did not have any symptoms post-procedure and was discharged. Another subject developed pneumonia $48 \mathrm{~h}$ post-procedure and was treated with oral antibiotics at home with resolution of 
Table 4 Biopsy outcomes

\begin{tabular}{ll}
\hline Variable & Outcome \\
\hline $\begin{array}{ll}\text { Biopsy completion, } n(\%)^{\mathrm{a}} \\
\text { Subject level }(\mathrm{n}=60)\end{array}$ & $58(96.7)$ \\
& $95 \% \mathrm{Cl}: 92.1 \%, 100 \%{ }^{\mathrm{b}}$ \\
Nodule level $(\mathrm{n}=67)$ & $65(97.0)$ \\
& $95 \% \mathrm{Cl}: 92.9 \%, 100 \%{ }^{\mathrm{b}}$ \\
Closest distance to nodule, median mm (IQR) & $7.0(2.0,12.0)$ \\
Serious adverse events, $\mathrm{n}(\%)$ & $0(0)$ \\
Pneumothorax (with or without chest tube) & $0(0)$ \\
Airway bleeding (with or without intervention) & $0(0)$ \\
Other & $0(0)$ \\
Complications, $\mathrm{n}(\%)$ & $2(3.3)$ \\
Cardiac arrhythmia, CTCAE grade 2 (intra-proce- & $1(1.7)$ \\
$\quad$ dure) & $1(1.7)$ \\
Pneumonia, CTCAE grade 2 (post-procedure) &
\end{tabular}

IQR interquartile range, $\mathrm{Cl}$ confidence interval

CTCAE Common Terminology Criteria for Adverse Events; from reference 11

a Biopsy completion = tool passed through catheter and a sample was obtained

${ }^{\mathrm{b}} 95 \%$ Confidence Interval calculated using the Wald method

${ }^{c}$ Closest distance from tip of catheter to edge of the virtual nodule

symptoms after approximately 10 days. Neither complication was attributed to the SSRAB system.

\section{Discussion}

Although the standard of care for the majority of incidentally found pulmonary nodules is active surveillance, any nodule greater than $8 \mathrm{~mm}$ with a moderate pretest probability potentially warrants biopsy [13]. The introduction of new technologies, such as ENB and rEBUS have advanced bronchoscopy, but despite these technologies and tools, the reported diagnostic yield for peripheral nodules $<2 \mathrm{~cm}$ is $40-67 \%[1,7,14,15]$.

The early data from pre-clinical studies and the singlecenter first human use experience with SSRAB suggest that this very stable and sensitive platform allows bronchoscopists to guide tools and localize peripheral tumors to a higher degree than was previously possible [2]. The lead-in results for the larger PRECIsE Study of the Ion Endoluminal System are the earliest cases performed at six centers by eleven physicians, including both interventional pulmonologists and thoracic surgeons. None of the physicians had used the SSRAB system prior to these cases, and this initial series was limited to the first 10 cases for each center or the first 5 cases each for centers with more than one bronchoscopist.

Small peripheral nodules, which historically have low diagnostic yields, were targeted. The SSRAB technology facilitated navigation to this type of nodule. Despite the introductory nature of the technology for the bronchoscopists, they approached nodules with a median axial size $<18 \mathrm{~mm}$. The ability to drive the catheter to the target and then fix the catheter in location allowed passage of rEBUS, a system-specific flexible biopsy needle, forceps, or brushes without deviation from the target. Significant in each case was the ability of the Flexision needle to traverse very tight angles into peripheral airways allowing for repeat sampling at the same location, as illustrated in Fig. 7. This initial experience suggests the investigators were able to target small peripheral lesions during the first few cases, expeditiously and safely; however, diagnostic sensitivity and diagnostic yield metrics associated with nodules of this size warrants further investigation. While the clinical utility of the system will be reported in future publications, in most cases, EBUS lymph node staging was performed after biopsy during the same procedure in order to potentially reduce CT-body divergence (single anesthetic event) optimizing workflow for comprehensive diagnosis of suspicious nodules with complete staging in a single procedure [16].

In an operating room or bronchoscopy suite, each minute is a unit of time that influences personnel use and direct costs. Our cases had a median procedure time of $66.5 \mathrm{~min}$ and total biopsy workflow time of $31.5 \mathrm{~min}$. Folch et al. recently reported data from a large multicenter evaluation of ENB, $92.2 \%$ of whom performed $>5$ ENB procedures per month with experienced ENB teams [7]. Their median total procedure time was $52 \mathrm{~min}$ and median total ENB-specific procedure time was $25 \mathrm{~min}$. Given the established teams and navigational platforms in their reported study, we are encouraged by our initial experience and anticipate comparable procedural times using the SSRAB system in the subsequent phase of the PRECIsE study.

Because the unique shape-sensing feedback (rather than electromagnetic navigation) is fundamental to the Ion system, biopsy tools can be introduced through the catheter and simultaneous use of fluoroscopy can be incorporated throughout the procedure without affecting navigational accuracy. The real-time feedback allows for frequent referencing to the virtual target throughout the procedure and does not require movement of equipment into and/or out of the procedure field, providing for continuity in procedural workflow and facilitating stability of the catheter when positioned for biopsy. The physician can potentially correct for perceived CT-to-body divergence during navigation by identifying airways on the camera image and subsequently comparing them to the virtual image. If the physician believes the catheter is in the wrong airway, alternative airways can be quickly identified and accessed. Similarly, the virtual nodule position can be adjusted for rEBUS and fluoroscopic input, allowing an organized, accurate biopsy procedure. 


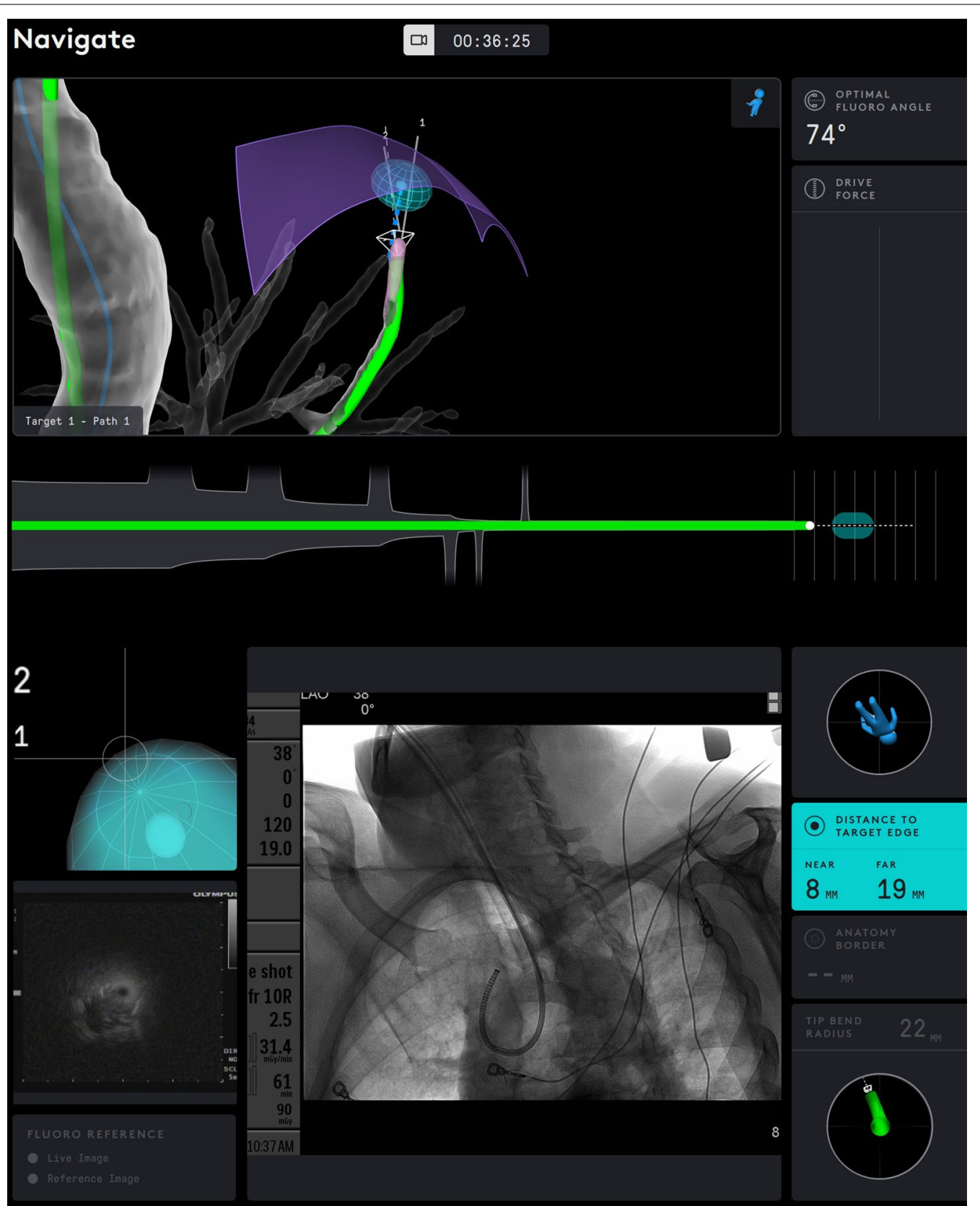

Fig. 7 Display of catheter on the system monitors during biopsy. The catheter position is shown in green and is interlaid on the virtual airway map. On the bottom screen, fluoroscopy reveals the tight bend of the catheter through the airways to reach the target nodule. Distance to the nearest and farthest edge of the target nodule is displayed at the lower right corner

With one-third of the nodules at least 8 generations out, the investigators perceived that SSRAB accessed nodules in peripheral locations. With the nodule identified, the combination of catheter size and flexibility, real-time shape feedback, and live visualization during navigation facilitated not only reaching within $7.0 \mathrm{~mm}$ of the virtual target but also passage of biopsy tools, including the Flexision biopsy needle, through any bend of the catheter to obtain tissue samples from the intended location. These outcomes suggest SSRAB will contribute significantly to the management of suspicious peripheral nodules.

Sampling, using the flexible needle, forceps, and/or brush biopsy tools, was performed with virtual targeting, fluoroscopy, and rEBUS. The shape-sensing technology detected and corrected for any catheter tip deflection 
that occurred from the insertion or removal of the rEBUS catheter or biopsy tools. By leveraging the capability to perform cloud biopsies, the broncoscopist can use the virtual, fluoroscopic, and ultrasound images to further localize the lesion for additional sampling, updating the position of the virtual target based on the gained realtime information. That the nodule visualization rate using rEBUS was $89.4 \%$ at the terminus of navigation demonstrates the initial localization success that SSRAB may provide, despite the majority of nodules not having a bronchus sign on pre-procedure CT. Early rEBUS literature reported visible lesions in approximately $67 \%$ of cases while more recent studies have reported an increase to $96 \%[17,18]$. rEBUS visualization characteristics and determination of true nodule identification versus artifact or atelectasis interpreted as nodule visualization is being studied [19]. Future studies may benefit from a standardized definition and review of imaging to confirm rEBUS visualization and characteristics.

Despite the high biopsy rate (96.7\%) in the current study, there were no events of pneumothorax or hemorrhage. This compares with the full cohort in the NAVIGATE study where the overall pneumothorax rate was $4.3 \%$ and, for those pneumothorax graded CTCAE $\geq 2$, the rate was $2.9 \%$. The ENB-related bronchopulmonary hemorrhage rate graded $\mathrm{CTCAE} \geq 2$ was $1.5 \%$ [7]. The encouraging safety profile should be noted in the context of this series describing the first human use of this technology for each bronchoscopist and suggests this technology's comparable safety profile to guided bronchoscopic approaches and significantly improved over percutaneous biopsy approaches. While encouraging, safety speculation is based on a limited number of subjects and further safety data from a larger cohort is forthcoming. As a remote-manipulator system, one of the limitations of robotic systems is the lack of haptic feedback that the physician may be accustomed to when performing manual bronchoscopy. During our initial experience, the use of visual cues provided feedback and confidence during navigation and sampling, supported by the low complication rate and the absence of observed airway trauma. Future publications will evaluate the sensitivity of malignancy and yield associated with this system relative to other approaches to further contextualize the value of Ion's safety profile.

Given the lead-in nature of this study, it has inherent limitations. All of the bronchoscopists and their teams were new to SSRAB technology in live cases and, thus, the described data were gained early in their learning curve. However, because they are highly experienced with bronchoscopic procedures, including with other navigation platforms, their experiences with SSRAB may not reflect the real world of bronchoscopists who may be early in their interventional pulmonary practice. As with any new technology, the use of SSRAB, its integration into the workflow, and the experience of the bronchoscopist and team all can affect durations and outcomes. Despite the fact that each center and physician had significant experience with ENB and other advanced diagnostic approaches, a standardized training program with mentorship provided by technical staff was completed prior to the first human experience. Performance metrics such as yield and sensitivity were not assessed due to limited follow-up, however performance metrics met the purpose of the lead-in phase. Further study is needed to evaluate performance. Other limitations include the enrollment of subjects according to controlled eligibility criteria, although the study's eligibility criteria modeled the population typically indicated for this type of biopsy procedure. Furthermore, the specific biopsy sequence and tool usage, as well as assessment of characteristics of CT bronchus sign or rEBUS visualization was not standardized: the view-eccentric or concentric-was determined by physician assessment. This was by design a goal of this lead-in stage to understand the real-world workflow and experience associated with this new technology. Last, the intent of the current single-arm analysis was generation of evidence regarding early experience. Future comparative studies should be considered in those centers where experience and proficiency with current technologies have been obtained. Strengths of the study are its multicenter design and the prospective collection and reporting of data from each bronchoscopist's first cases.

\section{Conclusions}

In this early experience with the Ion Endoluminal System and its shape-sensing navigation technology, bronchoscopists were successful in their ability to safely drive the catheter tip within close proximity of the virtual target for peripheral nodules, and they were able to obtain one or more biopsy samples of small, peripherally based nodules and-when necessary-perform lymph node staging within the same procedure. Both site and research-specific experiences have led to modified approaches and workflows with SSRAB, setting the stage for the full prospective evaluation, which will be reported at study completion. This initial, multicenter experience is encouraging and suggests the significant role SSRAB may play in the management of pulmonary nodules.

\section{Abbreviations}

CT: Computed tomography; CTCAE: Common terminology criteria for adverse event; ENB: Electromagnetic navigation bronchoscopy; EUS-B: Transesophageal ultrasound/endobronchial ultrasound; IQR: Interquartile range; IRB: Institutional review board; rEBUS: Radial endobronchial ultrasound; ROSE: 
Rapid on-site cytology evaluation; SSRAB: Shape-sensing robotic-assisted bronchoscopy; TBNA:Transbronchial needle aspiration.

\section{Acknowledgements}

The authors acknowledge members of the Intuitive Clinical Affairs team for study operation support; members of the Intuitive Clinical Development Engineering team and First Case Support teams for their guidance; and Wainwright Medical Communications (Los Gatos, CA) for editorial support.

\section{Authors' contributions}

All authors were involved in and contributed to the development of the study design and the collection and review of the data. MJS provided the initial draft of the manuscript. All authors critiqued and revised the initial and subsequent manuscript drafts and approved the final analysis and submitted manuscript files.

\section{Funding}

Study sponsored by Intuitive Surgical, Inc. for data collection, statistical analysis, and editorial support. Authors retained full control of the development, review, and final approval of the manuscript.

\section{Availability of data and materials}

The datasets generated and/or analyzed during the current study are not publicly available because the current study describes the initial analysis, which is not inclusive of the overall results of the PREClsE Study; the datasets are available from the corresponding author upon reasonable request.

\section{Declarations}

\section{Ethics approval and consent to participate}

Each center obtained institutional review board (IRB) (ethics) approval, and enrolled study participants provided written informed consent. Study subject confidentiality was maintained according to the Health Insurance Portability and Accountability Act requirements. All research was performed in accordance with the 1964 Declaration of Helsinki and its amendments. Study sites, IRB committee names, and approval numbers follow: Mayo Clinic: Mayo Clinic IRB 18-011348; First Health Moore Regional Hospital: Western IRB 20183121; Massachusetts General Hospital: Dana Farber Cancer Institute IRB 19-209; Beth Israel Deaconess Medical Center: Dana Farber Cancer Institute IRB 19-209; Henry Ford Hospital: Henry Ford Health System IRB 12822; University of Texas MD Anderson Cancer Center: MD Anderson Cancer Center IRB IRB00006023.

\section{Consent for publication}

Not applicable.

\section{Competing interests}

MJS has received research and manuscript support from Intuitive Surgical and consulting fees and research support from Gongwin Biopharm. MAP has received consulting and speaker fees and research support from Johnson \& Johnson, Medtronic, BodyVision, and Intuitive Surgical; research support from Philips; speaker fees and research support from Biodesix; consulting and speaker fees from AstraZeneca; speaker fees from Boeinger-Ingleheim; speaker fees and research support from United Therapeutics; speaker fees and research support from Actelion; research support from Inivata; consulting fees from Boston Scientific; consulting fees from OncoSec; consulting fees from Ambu; consulting fees and stock from HealthMyne. JSR has received research support and an educational grant from Intuitive Surgical. DEO has received research support, a research grant, and consulting fees from Intuitive Surgical. AM has received research support from Intuitive Surgical, consulting fees from Olympus America, and a research grant from Bronchus Medical. CK has received consulting fees and research support from Intuitive Surgical. RFC has received research support from Olympus, Concordia, Intuitive Surgical, and Siemens and consulting fees from Olympus, Siemens, Intuitive Surgical, Johnson \& Johnson, and Bronx. MSP has received research support and educational consultant fees from Intuitive Surgical. JD-M has received research support from Intuitive Surgical. SF-B has received research support from Intuitive Surgical. EEF has received a research grant from Intuitive Surgical and consulting fees from Boston Scientific and Medtronic.

\section{Author details}

${ }^{1}$ Bronchoscopy and Interventional Pulmonology, Lung Cancer Screening Program, Department of Pulmonary and Critical Care Medicine, Henry Ford Hospital, Wayne State University School of Medicine, 2799 West Grand Blvd, Detroit, MI 48202, USA. ${ }^{2}$ Pulmonary Department, Pinehurst Medical Clinic, Pinehurst, NC, USA. ${ }^{3}$ Pulmonary Department, First Health Moore Regional Hospital, Pinehurst, NC, USA. ${ }^{4}$ Department of Pulmonary Medicine and Thoracic Surgery, Mayo Clinic, Rochester, MN, USA. ${ }^{5}$ Department of Pulmonary Medicine, The University of Texas MD Anderson Cancer Center, Houston, TX, USA. ${ }^{6}$ Department of Thoracic Surgery and Interventional Pulmonology, Beth Israel Deaconess Medical Center, Harvard Medical School, Boston, MA, USA. ${ }^{7}$ Department of Pulmonary and Critical Care Medicine, Massachusetts General Hospital, Harvard Medical School, Boston, MA, USA. ${ }^{8}$ Department of Pulmonary Medicine, Mayo Clinic, Jacksonville, FL, USA.

Received: 21 January 2021 Accepted: 28 September 2021 Published online: 16 October 2021

\section{References}

1. Gould MK, Tang T, Liu IL, et al. Recent trends in the identification of incidental pulmonary nodules. Am J Respir Crit Care Med. 2015;192(10):1208-14. https://doi.org/10.1164/rccm.201505-09900C.

2. Wahidi MW, Govert JA, Goudar RK, Gould MK, McCrory DC, and The American College of Chest Physicians. Evidence for the treatment of patients with pulmonary nodules: When is it lung cancer? ACCP evidence-based clinical practice guidelines (2nd edition). Chest. 2007;132(3 Suppl):94s-107s. https://doi.org/10.1378/chest.07-1352.

3. Kalanjeri S, Holladay RC, Gildea TR. State-of-the-art modalities for peripheral lung nodule biopsy. Clin Chest Med. 2018;39:125-38. https://doi.org/ 10.1016/j.ccm.2017.11.007.

4. Baaklini WA, Reinoso MA, Gorin AB, Sharafkaneh A, Manian P. Diagnostic yield of fiberoptic bronchoscopy in evaluating solitary pulmonary nodules. Chest. 2000;117(4):1049-54. https://doi.org/10.1378/chest.117.4. 1049.

5. Wang Memoli JS, Nietert PJ, Silvestri GA. Meta-analysis of guided bronchoscopy for the evaluation of the pulmonary nodule. Chest. 2012;142(2):385-93.

6. Tanner NT, Yarmus L, Chen A, et al. Standard bronchoscopy with fluoroscopy vs thin bronchoscopy and radial endobronchial ultrasound for biopsy of pulmonary lesions: a multicenter, prospective, randomized trial. Chest. 2018;154(5):1035-43. https://doi.org/10.1016/j.chest.2018.08.1026.

7. Folch EE, Pritchett MA, Nead MA, et al. Electromagnetic navigation bronchoscopy for peripheral pulmonary lesions: one-year results of the prospective, multicenter NAVIGATE Study. J Thorac Oncol. 2019;14(3):445-58. https://doi.org/10.1016/j.tho.2018.11.013.

8. Folch EE, Labarca G, Ospina-Delgado D, et al. Sensitivity and safety of electromagnetic navigation bronchoscopy for lung cancer diagnosis: systematic review and meta-analysis. Chest. 2020. https://doi.org/10. 1016/j.chest.2020.05.534.

9. Fielding DIK, Bashirzadeh F, Son JH, et al. First human use of a new robotic-assisted fiber optic sensing navigation system for small peripheral pulmonary nodules. Respiration. 2019;98(2):142-50.

10. Swensen SJ, Silverstein MD, Ilstrup DM, Schleck CD, Edell ES. The probability of malignancy in solitary pulmonary nodules. Application to small radiologically indeterminate nodules. Arch Intern Med. 1997;157(8):849-55.

11. Folch EE, Mahajan AK, Oberg CL, et al. Standardized definitions of bleeding after transbronchial lung biopsy: a delphi consensus statement From the Nashville Working Group. Chest. 2020;158(1):393-400. https://doi.org/ 10.1016/j.chest.2020.01.036.

12. Du Rand IA, Blaikley J, Booton R, et al. for the British Thoracic Society Bronchoscopy Guideline Group. British Thoracic Society guideline for diagnostic flexible bronchoscopy in adults: accredited by NICE. Thorax. 2013;68(Suppl 1):1-44. https://doi.org/10.1136/thoraxjnl-2013-203618.

13. U.S. Department of Health and Human Services, National Institutes of Health, National Cancer Institute. Common Terminology Criteria for Adverse Events (CTCAE) version 5.0. November 27, 2017. Accessed September 25, 2020 
14. Gould MK, Donington J, Lynch WR, et al. Evaluation of individuals with pulmonary nodules: When is it lung cancer? Diagnosis and management of lung cancer. 3rd ed: American Collage of Chest Physicians evidence-based clinical practice guidelines. Chest. 2013(May suppl);143:e93s-e119s.

15. Ellis PM, Vandermeer R. Delays in the diagnosis of lung cancer. J Thorac Dis. 2011;3(3):183-8. https://doi.org/10.3978/j.issn.2072-1439.2011.01.01.

16. Pritchett MA, Bhadra K, Calcutt M, Folch E. Virtual or reality: divergence between preprocedural computed tomography scans and lung anatomy during guided bronchoscopy. J Thorac Dis. 2020;12(4):1595-611. https:// doi.org/10.21037/jtd.2020.01.35.

17. Eberhardt R, Ernst A, Herth FJF. Ultrasound-guided transbronchial biopsy of solitary pulmonary nodules less than $20 \mathrm{~mm}$. Eur Respir J. 2009;34(6):1284-7. https://doi.org/10.1183/09031936.00166708.
18. Chen A, Chenna P, Loiselle A, Massoni J, Mayse M, Misselhorn D. Radial probe endobronchial ultrasound for peripheral pulmonary lesions. A 5-year institutional experience. Ann Am Thorac Soc. 2014;11(4):578-82. https://doi.org/10.1513/AnnalsATS.201311-384OC.

19. Sagar A-ES, Sabath BF, Eapen GA, et al. Incidence and location of atelectasis developed during bronchoscopy under general anesthesia: the I-LOCATE trial. Chest. 2019. https://doi.org/10.1016/j.chest.2020.05.565.

\section{Publisher's Note}

Springer Nature remains neutral with regard to jurisdictional claims in published maps and institutional affiliations.
Ready to submit your research? Choose BMC and benefit from:

- fast, convenient online submission

- thorough peer review by experienced researchers in your field

- rapid publication on acceptance

- support for research data, including large and complex data types

- gold Open Access which fosters wider collaboration and increased citations

- maximum visibility for your research: over $100 \mathrm{M}$ website views per year

At BMC, research is always in progress.

Learn more biomedcentral.com/submissions 